\title{
El concepto de calidad de vida en las teorías del desarrollo ${ }^{1}$
}

\section{The concept of quality of life in the theories of development}

\author{
BÁRbARA PATRICIA ARIAS CORTÉS ${ }^{2}$ \\ bpattyarco@hotmail.com
}

\section{RESUMEN}

Este documento plantea un análisis del concepto de calidad de vida que se ha expuesto en las teorías del desarrollo. A efecto de lo señalado, en primer lugar se revisará lo que se ha entendido por desarrollo y a partir de ello, en un segundo momento, se analizará cuál es el concepto de calidad de vida inmerso en teorías tales como la del desarrollo a partir de capacidades, el desarrollo a escala humana y el desarrollo humano sostenible.

Lo anterior refleja la manera como han evolucionado los dos conceptos (tanto el de desarrollo como el de calidad de vida), conduciendo a lo que hoy se plantea como corriente alternativa en algunos países de América Latina, y que se conoce como buen vivir.

PALABRAS CLAVE: desarrollo, desarrollo humano, calidad de vida, alternativas para el desarrollo.

Fecha de recepción: marzo 11 de 2013

Fecha de aceptación: abril 1 de 2013

\section{ABSTRACT}

This paper presents an analysis of the concept of quality of life that has been exposed in developmental theories. The effect of the above, is first reviewed what has been understood by development and beyond that, in a second phase, which will be discussed, is the concept of quality of life immersed in theories such as Developmental Capacities, from the Human Scale Development and Sustainable Human Development.

This reflects how the two concepts have evolved (both in the development and in the quality of life), leading to what is now considered an alternative trend in some Latin American countries, and is known as Good Live.

KEYWORDS: development, human development, quality of life, development alternatives.

1. Artículo de reflexión resultado de la investigación adelantada dentro del programa Teorías y enfoques de los derechos humanos. Nuevos enfoques y perspectivas diferenciales de los derechos humanos, Maestría en Derechos Humanos de la Universidad UPTJ (sede Chiquinquirá).

2. Abogada, especialista en Derecho Laboral y Seguridad Social, estudiante de maestría en Derechos Humanos de la Universidad Pedagógica y Tecnológica de Colombia. Docente de la Escuela de Administración de Empresas de la UPTC (Chiquinquirá). Adscrita al grupo de investigación “Grupo de Estudios Regionales Julio Flórez”.

Criterio Jurídico Garantista. AÑo 5, No. 8. Ene.-Jun. de 2013. ISSN: 2145-3381. Fundación Universidad Autónoma de Colombia, Bogotá. 


\section{Introducción}

El crecimiento de los países se mide por las condiciones de vida de sus habitantes. Dichas condiciones, junto con otros elementos, inciden también en la calidad de vida de los sujetos, por lo que se ha convertido en interés constante de los gobiernos el formular políticas públicas tendientes a mejorar tanto las condiciones de vida como la calidad de esta para generar, en últimas, mayor desarrollo de las capacidades.

En Colombia, las políticas públicas de manera generalizada se formulan bajo sistemas de medición cuantitativa, por cuanto en buena manera esas políticas se fundamentan en los resultados de instrumentos como la Encuesta Nacional de Calidad de Vida (ENCV), aplicada por el Departamento Administrativo Nacional de Estadística (DANE), que no comportan una visión multidisciplinar del desarrollo, de manera que el mejoramiento de la calidad de vida por aspectos vinculados al desarrollo humano presentan dificultades para su implementación.

Conforme a lo anterior, se plantea analizar el concepto de cali- dad de vida en el marco de las diferentes teorías del desarrollo y cómo la noción del desarrollo se va ampliando, más allá de las simples mediciones del ingresogasto de los sujetos y los países, para avanzar hacia enfoques que contemplan tanto la calidad de vida como el desarrollo de otros componentes que comportan la propia condición humana.

Abordaremos el estudio de algunas nociones que comprenden la calidad de vida a partir de las capacidades, el desarrollo a escala humana y el desarrollo humano sostenible, para continuar con el análisis de dicha noción dentro de algunas de las corrientes de formación más recientes como la del "buen vivir", asumidas como propuestas alternativas que se están implementando en Bolivia, Ecuador y Colombia, $\mathrm{y}$, finalmente, las conclusiones.

\section{Metodología}

El presente escrito plantea un análisis documental comparativo de las teorías del desarrollo y el concepto de calidad de vida, el pensamiento de Amartya Sen con relación al desarrollo y el concepto de calidad de vida con el resultado de la Encuesta Na- cional de Calidad de Vida, que en el caso colombiano sirve de base para la formulación de políticas públicas, de ahí la necesidad de reconsiderar la noción y los lineamientos de política pública, que en Colombia sostiene una visión tradicional a pesar de que los informes de la ONU exigen introducir en los planes de desarrollo el enfoque en capacidades.

Los hallazgos de la contrastación enunciada nos permitieron realizar el análisis y examinar si el enfoque multidisciplinar de desarrollo propuesto por algunos autores se cumple en el marco de la obligatoriedad del Estado de promover y generar planes de acción que consideren el desarrollo humano como eje relevante de los planes de desarrollo y del mejoramiento de la calidad de vida de las poblaciones, teniendo en cuenta factores que vayan más allá de la medición cuantitativa de ingresos materiales per cápita.

\section{Desarrollo y calidad de vida}

Comprender la importancia de conocer e implementar el enfoque de desarrollo desde una visión integral requiere abordar diferentes aspectos que inciden 
en él y que son de orden económico, social, político, cultural e histórico.

Para alcanzar el propósito planteado es preciso referir qué se entiende por desarrollo, a efecto de lo cual Eduardo Gudynas (2012) ha expuesto que:

Los sentidos usuales de la palabra desarrollo apuntan a los avances y progresos en el campo económico y social. En ese sentido la Real Academia Española presenta el desarrollo como una acepción económica entendida como la "evolución progresiva de una economía 138 hacia mejores niveles de vida", mientras que, cuando se lo refiere a las personas, se lo define como progreso, bienestar, modernización, crecimiento económico, social, cultural o político.

Lo anterior implica que el desarrollo, desde el campo meramente económico, puede referirse o asociarse con el concepto de "avance", entendido como el mejoramiento del nivel social o de las condiciones de subsistencia propias de un individuo o comunidad, y a la vez comprender elementos que van más allá del deseo y de la percepción personal, y que por ende trascienden al entorno del individuo.
Conforme a lo señalado, el concepto de desarrollo ha sufrido cambios que han estado ligados a las transformaciones propias de las sociedades, como lo deja ver, por ejemplo, el Informe sobre Desarrollo Humano de 1994 en el que se plantea que inicialmente:

El concepto de desarrollo trataba el ingreso y su crecimiento como un medio y dirigía la atención a una preocupación auténtica por la gente, en forma individual y colectiva, sus rasgos en común y su diversidad. La preocupación central del desarrollo pasó a ser la calidad de la vida de las personas, lo que eran capaces de hacer y lo que hacían efectivamente, la discriminación que enfrentaban, las luchas que libraban $y$ las crecientes opciones de las que gozaban. (PNUD, 1994, p. 16).

El enfoque de desarrollo humano, al que se hace alusión en el informe referido, ha continuado su expansión. Por ejemplo, en el reciente informe denominado “Un Sur más Global”, se resalta cómo en algunos de los llamados países en desarrollo, entre los que se cuentan Argelia, Argentina, Brasil y Bangladés, al priorizar a las personas en sus planes de desarrollo, mejora su inserción en el mundo global (PNUD, 2013, p. 43). Es decir, el desarrollo humano se convierte en un proceso para ampliar las oportunidades de las personas, tales como la de prolongación de la vida y de la salud, el acceso a la educación y a disfrutar una vida en condiciones dignas y una vida con calidad.

Como afirma Sen (2009, pp. 19, 35 ), el desarrollo es un proceso de expansión de las libertades reales de que disfrutan los individuos. Afirma el autor que el aumento de la libertad mejora las capacidades del individuo para apoyarse en el desarrollo de su propio plan de vida y para influir en el mundo. Un conjunto de oportunidades que se liga con las capacidades propias de los individuos, como criterios a tener en cuenta para evaluar el bienestar y la calidad de vida. Esta noción fue introducida en el contexto de la teoría de la justicia y ha sido asumida y utilizada ampliamente por las Naciones Unidas para conceptualizar primero y medir después el desarrollo humano.

En el sentido enunciado, la libertad es una razón para evaluar el progreso en función del aumento que esta haya experimentado y de la libertad de agenciar que adquieren y realizan los indivi- 
duos como sujetos de cambio, cuyos logros pueden medirse en función de sus propios valores y objetivos. Este enfoque del desarrollo humano considera factores sociales, familiares, laborales y culturales que inciden directamente en el individuo y que hacen parte, por consiguiente, de un componente muchísimo más importante: el enfoque de desarrollo a partir de lo que puede o es"capaz" de hacer una persona.

La conjunción que existe entre esos dos conceptos (desarrollo y capacidades humanas) ha sido expuesta también por Martha Nussbaum, quien señala que:

El enfoque de las capacidades tiene otro uso, afín y de menor relevancia. El mismo determina un espacio dentro del cual las comparaciones de calidad de vida (cómo de bien le va a la gente), cuando se las establece entre las distintas naciones, resultan más reveladoras. Al utilizarlo de este modo, este enfoque rivaliza con otras mediciones estándar como el PIB per cápita y la utilidad. (Nussbaum, 2012, p. 33).

El enfoque de desarrollo a partir de capacidades, ya expuesto, se aleja de las mediciones de la calidad de vida ligadas a fríos porcentajes, pues va más allá, en el sentido de incluir diferentes factores, no tenidos en cuenta antes, y que inciden en la vida de los seres humanos, donde lo verdaderamente importante no es cuánto posea el individuo (hablando en términos de recursos económicos), sino más bien qué es capaz de hacer, cómo puede por sus propios medios mejorar su calidad de vida y qué le puede aportar a la sociedad.

Los planteamientos señalados han servido de fundamento al concepto de desarrollo expuesto en los Informes del Programa de las Naciones Unidas para el Desarrollo (PNUD), pues como lo indica Montesino (2001), a Sen se le ha considerado "uno de los precursores o inspiradores del llamado índice de desarrollo humano (IDH)”, el cual incorpora además del ingreso de la población de un país un conjunto de factores tales como la educación, la salud, la seguridad, la descentralización y la no discriminación por género.

Lo anterior simplemente reafirma el argumento respecto de la complementariedad de factores que inciden en el desarrollo de los seres humanos.

Siguiendo en la misma línea, Sen señala que "la perspectiva basada en la libertad guarda una similitud genérica con la preocupación habitual por la [calidad de vida], que también centra la atención en la forma en que transcurre la vida humana" (Sen, 2000, p. 42).

La calidad de vida, conforme la define el autor citado, implica en primera medida "tener buenas condiciones de vida 'objetivas' y un alto grado de bienestar 'subjetivo', e incluye también la satisfacción colectiva de necesidades a través de políticas sociales" (Palomba, 2009, p. 3).

De acuerdo con lo señalado, se considera que el desarrollo económico pugna con el desarrollo humano, pues como indica el profesor Lampis (2007, p. 34): "la concepción basada en la idea de crecimiento económico, centrada en políticas instrumentales y guiada por la disciplina económica, se enfrenta a otra que aboga por la multidimensionalidad de aquel, la necesidad de su comprensión interdisciplinaria y su orientación hacia el desarrollo humano”.

Lo expuesto a propósito del desarrollo y la calidad de vida conlleva ligar su concepto con el de bienestar, satisfacción de 
necesidades propias e incluso con el de felicidad, es decir, que comporta una gama importante de subjetividades que se desprenden de todo aquello que está interconectado con el sujeto.

Para especificar más aún, Armando de Negri (2002, p. 2) nos plantea de la siguiente forma la noción de calidad de vida:

se asocia con lo que se valora socialmente como bueno o deseable, y también con lo que hace movilizar energías, recursos y esfuerzos para que 140 sea efectivamente alcanzado en un punto del futuro. Es un concepto integrador de las necesidades humanas que rompe con las concepciones fragmentadas y parciales de la realidad social y desarrolla un enfoque continuo de la vida y de sus determinaciones sociales en todas sus etapas, procesos $\mathrm{y}$ contextos. Propone igualmente formas de construcción de respuestas colectivas que trascienden las políticas centradas en la prestación de servicios y articula las políticas económicas y sociales alrededor de objetivos y metas públicas dirigidas a impactar en la transformación de las condiciones de vida de la gente.

La calidad de vida, en consecuencia, implica la conjunción de ideales, propósitos, necesidades básicas y recursos, que se constituyen en las realidades y contextos en que las personas habitan y construyen sus espacios de relaciones y que sirven de referentes comparativos respecto de otros sujetos. Al respecto continúa De Negri:

En este sentido, la calidad de vida representa dentro de una perspectiva política que busca la universalidad de los derechos sociales con equidad, que todos/as tengan igualdad de acceso e igualdad de oportunidades al pleno desarrollo y preservación de la autonomía como seres humanos y ciudadanos según las necesidades de cada quien, en forma individual y colectiva. Este pensamiento exige el compromiso político de construir una ciudadanía social o de derechos sociales y rescatar la función sustantiva de las políticas públicas en resguardo del interés colectivo.

Los elementos que orientan estas políticas públicas son:

- El imperativo ético de responder a las necesidades sociales con universalidad y equidad, cerrando los déficits de necesidades sociales y reduciendo las inequidades en las condiciones de calidad de vida.

- La confluencia de esfuerzos colectivos dirigidos a preservar y desarrollar la autonomía de individuos y colectividades en el ejercicio de los derechos sociales y la plena realización de la vida.

- La reorientación del modo de producir respuestas haciéndolas regulares, suficientes, integrales y equitativas, a través de redes públicas y de solidaridad social.

- La construcción de una nueva institucionalidad pública comprometida con la transformación de los patrones de calidad de vida de la sociedad (pp. 3-4).

En los términos expuestos se evidencia que el concepto de calidad de vida requiere del enfoque de derechos o enfoques de desarrollo dados a partir de esta base, en la medida que se hace necesario generar mayores y mejores oportunidades para los individuos; adicionalmente, con ello se contrae un serio compromiso por parte de los gobiernos para materializar políticas públicas que satisfagan verdaderamente las necesidades de los sujetos, y que no se queden en el simple análisis desde la visión económica, limitada a medir la relación ingreso-gasto. 
En el punto de los compromisos estatales frente al desarrollo surge una controversia entre discurso y realidad, pues en la práctica no se aprecia claramente que las políticas públicas apunten a mejorar la calidad de vida de los individuos, ya que muchas veces su interés se centra en factores o sectores que no son precisamente el foco del problema, como lo plantea $\mathrm{Mu}$ hhamad Yunus (1998, p. 263) cuando señala que la expansión demográfica descontrolada de algunos países pobres puede incidir en aumentar la pobreza, pero así mismo reconoce que implicados en los problemas demográficos hacen muchos menos esfuerzos por mejorar "los gobiernos y los organismos

la calidad de la vida que por aterrorizar a los pobres y a los analfabetos y presionarlos para que procreen menos".

Lo anterior implica que se está tratando de dar solución al problema bajo una óptica inadecuada, pues al tenor de lo planteado por Yunus, la pobreza no desaparecerá haciendo que las familias tengan menos hijos, sino que por el contrario, se podrá reducir en la medida en que sus integrantes puedan tener acceso a la educación, mejores oportunidades laborales y mejor remuneración.

Algo similar sucede en Colombia, como lo ejemplifica Lampis (2007, p. 77):

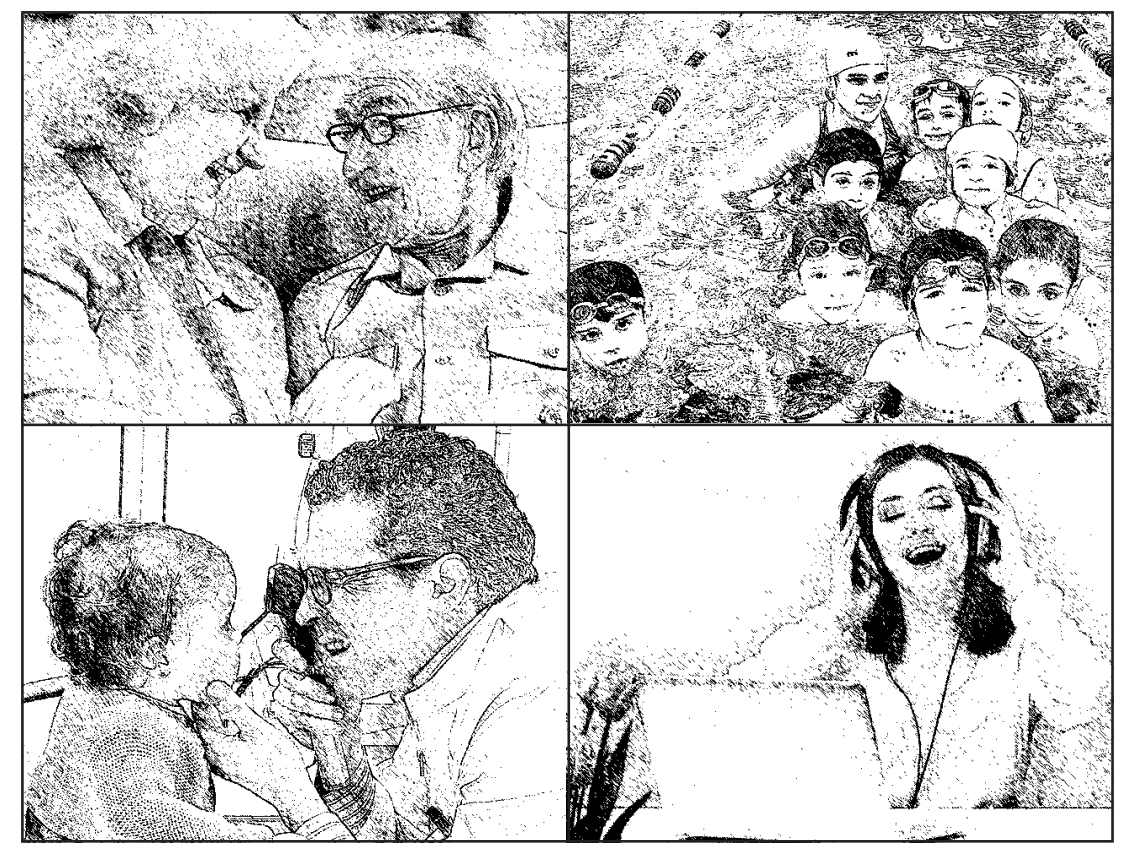

En el ámbito colombiano la investigación, soportada por la literatura nacional, indica que (...) No hay avances significativos en la mejora de la calidad de vida en el largo plazo desde 1997, lo que contrasta con los avances "ocasionales", en disminución de la pobreza por ingreso no se ha avanzado en reducir la pobreza desde una perspectiva multidimensional, como lo demuestra el grave atraso que se registra en cuanto a los Objetivos del Milenio en la mayoría de los departamentos y municipios del país.

Lo señalado por el autor pone en evidencia la falta de políticas públicas adecuadas, que verdaderamente apunten a un desarrollo basado en la satisfacción de necesidades del ser humano

La calidad de vida, en consecuencia, implica la conjunción de ideales, propósitos, necesidades básicas y recursos, que se constituyen en las realidades y contextos en que las personas habitan y construyen sus espacios de relaciones y que sirven de referentes comparativos respecto de otros sujetos. 
y del aumento de capacidades y de su promoción propia como agente de cambio.

Lo expuesto puede deberse en alguna medida a que el diseño, seguimiento y evaluación de las políticas públicas se basan de una u otra forma en los resultados de la ENCV que ha aplicado en distintas oportunidades en Colombia el DANE, encuesta que sigue fincada en aspectos que no miden realmente las capacidades de los sujetos.

Como lo señala el propio DANE

142 (2010), la ENCV ha surgido más bien como:

respuesta a la necesidad de caracterizar la población en los diferentes aspectos involucrados en el bienestar de los hogares. En 1986 y con el auspicio de las Naciones Unidas, DNP y UNICEF en el DANE se crea el proyecto ISPA (indicadores de pobreza absoluta), cuya labor fundamental consiste en cuantificar los pobres, caracterizarlos y ubicarlos espacialmente. Para ello se definen dos metodologías: la primera mide pobreza estructural, denominada necesidades básicas insatisfechas (NBI) y la segunda que mide pobreza coyuntural, denominada línea de pobreza (LP).
Como se puede colegir de lo citado, este instrumento, (la ENCV), no está diseñado sobre el enfoque multidimensional que indica Lampis, y contiene indicadores basados en preguntas que pueden ser muy subjetivas y que a la final pueden terminar no midiendo nada relevante en términos verdaderos de desarrollo y de capacidades, como se verá más adelante.

Asimismo, el eje de la mencionada ENCV es el índice de condiciones de vida (ICV), que permite "una aproximación a los perfiles de calidad de vida de los hogares y a la incidencia, brecha e intensidad, de la pobreza en ellos. El ICV, es un índice continuo que va de 0 a 100 , donde o refleja las peores condiciones de vida y 100 las mejores condiciones de vida. Un aumento del ICV indica una disminución de la pobreza” (Kalipedia, 2008).

Para el caso específico de Colombia, como indican Gamboa, Cortés y González (2000, pp. 36-37) el ICV resulta de aplicar la metodología de componentes principalmente de tipo cualitativo a la ENCV. La metodología contiene 12 variables agrupadas en 4 factores, que analiza, por ejemplo, materiales de los qué está hecha la vivienda, método de abastecimiento de agua, combustible con el que se cocina, etc.

El ICV, como señala Sarmiento (2010), en un comienzo le apuntaba a una visión multidimensional de la calidad de vida y a la vez a tener en cuenta aspectos no utilizados como capital humano y los diferentes grados de desarrollo, pero como ya se señaló, la ENCV no ha incluido del todo tal concepto de multidimensionalidad y algunas de las preguntas que contiene poco pueden medir en relación directa con el desarrollo de las personas y de sus capacidades.

Esta situación se puede apreciar en la práctica con el siguiente ejemplo: uno de los indicadores de la ENCV, efectuada en el año 2011 , era el de la "percepción de pobreza” de los encuestados respecto de sí mismos, para efecto de lo cual se les preguntaba "si se considera pobre".

De acuerdo con el resultado, el porcentaje de pobreza pasó del $44.4 \%$ en 2010 al $43.2 \%$ en 2011 , pero el asunto no se puede reducir simplemente a una cuestión numérica, ya que con base en las cifras lo que podría argüirse es que la pobreza se redujo en 
un $1.2 \%$, pero de la forma en que está diseñada la pregunta, netamente lo que ha variado es la "percepción de pobreza" mas no la situación real de pobreza del individuo.

Cabe cuestionarse si cuando una persona no se considera pobre o se considera menos pobre de lo que era antes, realmente su situación de pobreza ha disminuido o desaparecido. La respuesta puede ser tan subjetiva como la percepción misma de pobreza, pues para algunos puede ser pobre quien no tenga determinados ingresos o no posea ciertos bienes, para otros la pobreza puede estar ligada a la imposibilidad de sostener cierto "nivel de vida" o comodidades, y para otros de corte más hedonista, la pobreza puede estar más bien relacionada con situaciones que impidan alcanzar la felicidad.

En tal sentido, y a partir de semejante cuestionamiento, es bastante difícil determinar la situación concreta de pobreza de los encuestados y establecer un índice de medición coherente con la situación real de estos.

Otro aspecto que se analiza en la ENCV y que nos puede servir de ejemplo para demostrar que dicho instrumento no aplica el enfoque multidimensional, $\mathrm{y}$ que difícilmente mide la verdadera situación de las personas, es el referente a la educación, en el que rápidamente se concluye que la tasa de analfabetismo disminuyó 0.5 puntos porcentuales en 2011 con relación al 2010, lo cual además de no ser un avance muy significativo hace un llamado, más bien, a que se fortalezca la cobertura y calidad del sistema educativo colombiano, con una mayor y mejor inversión de recursos.

Siguiendo por el mismo orden, la ENCV 2011 revela, respecto de la cobertura de servicios públicos domiciliarios, que en acueducto, alcantarillado, gas natural y energía eléctrica se presentó aumento, mientras que en telefonía fija hubo una disminución en el número de usuarios, comparado con el año 2010.

En cuanto a la afiliación al Sistema General de Seguridad Social en Salud, la encuesta plantea que con relación al 2010, en el 2011 hubo un aumento de 1.4 puntos porcentuales en la afiliación, y si se analiza por regímenes, el contributivo presentó un incremento de 2.6 puntos porcentuales, mientras que el subsidiado registró una reducción de 2.3 puntos porcentuales.

Lo anterior permitiría concluir que en el 2011 un mayor número de población tuvo acceso a servicios públicos domiciliarios y prestación de servicios de salud, frente al 2010, pero lo que la encuesta no revela es en qué condiciones se están prestando esos servicios o a qué factores obedecen los cambios porcentuales, con lo que se puede afirmar que la ENCV sigue aún enfocada en la medición del desarrollo en términos puramente de cifras, pero no está midiendo a fondo la realidad de las comunidades.

Si bien es cierto que para muchos podrán ser alentadoras las cifras referentes al aumento de cobertura en servicios públicos domiciliarios y al sistema de salud, esto no necesariamente implica que en la realidad se esté mejorando la calidad de vida de la población, pues tanto el servicio de educación como el de salud en Colombia siguen siendo bastante deficientes. De los resultados expuestos podría decirse, entonces, que hay mayor cobertura pero no necesa- 
riamente una mejor prestación de dichos servicios.

\section{Resultados}

El análisis de la encuesta frente a la calidad de vida, su interacción con el enfoque basado en la expansión de las capacidades y su medición como centro del enfoque del desarrollo a partir de las aptitudes y derechos de las personas, implica que los sujetos sean vistos como algo más que cifras en unos índices, por cuanto se requiere considerar todos los factores tanto internos como externos que inciden en la calidad de vida de las personas. Así por ejemplo, Olga Berríos (2007) en el artículo El desarrollo a escala humana de Manfred Max-Neef, afirma que la propuesta de este autor tiene como protagonista básico a las personas y está sustentada en tres aspectos fundamentales: “a)
La satisfacción de las necesidades humanas. b) La generación de niveles crecientes de autodependencia. Esto se refiere a economías locales y regionales y c) La articulación orgánica entre seres humanos, naturaleza y tecnología”.

Para comprender de mejor forma lo anterior, veamos la matriz de necesidades y satisfactores que plantea Max-Neef:

\begin{tabular}{|c|c|c|c|c|}
\hline \multirow{2}{*}{$\begin{array}{l}\text { NECESIDADES } \\
\text { SEGÚN } \\
\text { CATEGORÍAS } \\
\text { AXIOLÓGICAS }\end{array}$} & \multicolumn{4}{|c|}{ NECESIDADES SEGÚN CATEGORÍAS EXISTENCIALES } \\
\hline & SER & TENER & HACER & ESTAR \\
\hline SUBSISTENCIA & $\begin{array}{l}\text { 1) Salud física, salud } \\
\text { mental, equilibrio, } \\
\text { sentido del humor, } \\
\text { adaptabilidad. }\end{array}$ & $\begin{array}{l}\text { 2) Alimentos, cobijo, } \\
\text { trabajo. }\end{array}$ & $\begin{array}{l}\text { 3) Alimentarse, pro- } \\
\text { crear, descansar, tra- } \\
\text { bajar. }\end{array}$ & $\begin{array}{l}\text { 4) Entorno vital, } \\
\text { marco social. }\end{array}$ \\
\hline PROTECCIÓN & $\begin{array}{l}\text { 5) Asistencia, adap- } \\
\text { tabilidad, autonomía, } \\
\text { equilibrio, solidari- } \\
\text { dad. }\end{array}$ & $\begin{array}{l}\text { 6) Sistemas de segu- } \\
\text { ros, ahorros, seguri- } \\
\text { dad social, sistemas } \\
\text { sanitarios, derechos, } \\
\text { familia, trabajo. }\end{array}$ & $\begin{array}{l}\text { 7) Cooperar, prevenir, } \\
\text { planificar, ocuparse } \\
\text { de curar, ayudar. }\end{array}$ & $\begin{array}{l}\text { 8) Espacio vital, en- } \\
\text { torno social, vivien- } \\
\text { da. }\end{array}$ \\
\hline AFECTO & $\begin{array}{l}\text { 9) Autoestima, de- } \\
\text { cisión, generosidad, } \\
\text { receptividad, pasión, } \\
\text { sensualidad, sentido } \\
\text { del humor, tolerancia, } \\
\text { solidaridad, respeto. }\end{array}$ & $\begin{array}{l}\text { 10) Amistades, re- } \\
\text { laciones familiares, } \\
\text { relaciones con la na- } \\
\text { turaleza. }\end{array}$ & $\begin{array}{l}\text { 11) Hacer el amor, } \\
\text { acariciar, expresar } \\
\text { emociones, compar- } \\
\text { tir, ocuparse de cul- } \\
\text { tivar, apreciar. }\end{array}$ & $\begin{array}{l}\text { 12) Vida privada, in- } \\
\text { timidad, hogar, espa- } \\
\text { cios de unión entre } \\
\text { personas. }\end{array}$ \\
\hline
\end{tabular}




\begin{tabular}{|c|c|c|c|c|}
\hline $\begin{array}{l}\text { NECESIDADES } \\
\text { SEGÚN } \\
\text { CATEGORÍAS }\end{array}$ & \multicolumn{4}{|c|}{ NECESIDADES SEGÚN CATEGORÍAS EXISTENCIALES } \\
\hline & SER & TENER & HACER & ESTAR \\
\hline COMPRENSIÓN & $\begin{array}{l}\text { 13) Conciencia crí- } \\
\text { tica, receptividad, } \\
\text { curiosidad, asombro, } \\
\text { disciplina, intuición, } \\
\text { racionalidad. }\end{array}$ & $\begin{array}{l}\text { 14) Literatura, maes- } \\
\text { tros, método, políti- } \\
\text { cas educativas, políti- } \\
\text { cas de comunicación. }\end{array}$ & $\begin{array}{l}\text { 15) Investigar, estu- } \\
\text { diar, experimentar, } \\
\text { educar, analizar, me- } \\
\text { ditar. }\end{array}$ & $\begin{array}{l}\text { 16) Marcos de inte- } \\
\text { racción formativa, es- } \\
\text { cuelas, universidades, } \\
\text { grupos, comunidades, } \\
\text { familia. }\end{array}$ \\
\hline PARTICIPACIÓN & $\begin{array}{l}\text { 17) Adaptabilidad, } \\
\text { receptividad, solida- } \\
\text { ridad, disposición, } \\
\text { decisión, dedicación, } \\
\text { respeto, pasión, sen- } \\
\text { tido del humor. }\end{array}$ & $\begin{array}{l}\text { 18) Derechos, res- } \\
\text { ponsabilidades, de- } \\
\text { beres, privilegios, } \\
\text { trabajo. }\end{array}$ & $\begin{array}{l}\text { 19) Afiliarse, coope- } \\
\text { rar, proponer, com- } \\
\text { partir, disentir, obe- } \\
\text { decer, relacionarse, } \\
\text { estar de acuerdo, ex- } \\
\text { presar opiniones. }\end{array}$ & $\begin{array}{l}\text { 20) Marcos de rela- } \\
\text { ciones participativas, } \\
\text { partidos, asociacio- } \\
\text { nes, Iglesias, comu- } \\
\text { nidades, barrios, fa- } \\
\text { milia. }\end{array}$ \\
\hline CREACIÓN & $\begin{array}{l}\text { 21) Pasión, decisión, } \\
\text { intuición, imagina- } \\
\text { ción, audacia, racio- } \\
\text { nalidad, inventiva, } \\
\text { autonomía, curiosi- } \\
\text { dad. }\end{array}$ & $\begin{array}{l}\text { 22) Habilidades, ofi- } \\
\text { cios, trabajo. }\end{array}$ & $\begin{array}{l}\text { 23) Trabajar, inven- } \\
\text { tar, construir, dise- } \\
\text { ñar, componer, inter- } \\
\text { pretar. }\end{array}$ & $\begin{array}{l}\text { 24) Marcos produc- } \\
\text { tivos y de reaprove- } \\
\text { chamiento de infor- } \\
\text { mación, seminarios, } \\
\text { grupos culturales, } \\
\text { espacios para la ex- } \\
\text { presión, libertad tem- } \\
\text { poral. }\end{array}$ \\
\hline RECREO & $\begin{array}{l}\text { 25) Curiosidad, senti- } \\
\text { do del humor, recep- } \\
\text { tividad, imaginación, } \\
\text { temeridad, tranquili- } \\
\text { dad, sensualidad. }\end{array}$ & $\begin{array}{l}\text { 26) Juegos, espectá- } \\
\text { culos, clubes, fiestas, } \\
\text { paz mental. }\end{array}$ & $\begin{array}{l}27 \text { ) Divagar, abs- } \\
\text { traerse, soñar, año- } \\
\text { rar, fantasear, evocar, } \\
\text { relajarse, divertirse, } \\
\text { jugar. }\end{array}$ & $\begin{array}{l}\text { 28) Privacidad, in- } \\
\text { timidad, espacios de } \\
\text { encuentro, tiempo } \\
\text { libre, ambientes, pai- } \\
\text { sajes. }\end{array}$ \\
\hline IDENTIDAD & $\begin{array}{l}\text { 29) Sentimiento de } \\
\text { pertenencia, consis- } \\
\text { tencia, diferenciación, } \\
\text { autoestima, afirma- } \\
\text { ción. }\end{array}$ & $\begin{array}{l}\text { 30) Símbolos, lengua- } \\
\text { je, religión, hábitos, } \\
\text { costumbres, grupos } \\
\text { de referencia, sexua- } \\
\text { lidad, valores, nor- } \\
\text { mas, memoria his- } \\
\text { tórica, trabajo. }\end{array}$ & $\begin{array}{l}\text { 31) Comprometerse, } \\
\text { integrarse, enfren- } \\
\text { tarse, decidir, cono- } \\
\text { cerse a uno mismo, } \\
\text { reconocerse a uno } \\
\text { mismo, realizarse, } \\
\text { crecer. }\end{array}$ & $\begin{array}{l}\text { 32) Ritmos sociales, } \\
\text { marcos de la vida } \\
\text { diaria, ámbitos de } \\
\text { pertenencia, etapas } \\
\text { de madurez. }\end{array}$ \\
\hline LIBERTAD & $\begin{array}{l}\text { 33) Autonomía, au- } \\
\text { toestima, decisión, } \\
\text { pasión, afirmación, } \\
\text { amplitud de miradas, } \\
\text { audacia, rebeldía, to- } \\
\text { lerancia. }\end{array}$ & $\begin{array}{l}34 \text { ) Igualdad de de- } \\
\text { rechos. }\end{array}$ & $\begin{array}{l}\text { 35) Discrepar, ele- } \\
\text { gir, ser diferente de, } \\
\text { asumir riesgos, de- } \\
\text { sarrollar conciencia, } \\
\text { comprometerse, des- } \\
\text { obedecer, }\end{array}$ & $\begin{array}{l}\text { 36) Plasticidad espa- } \\
\text { cio-temporal. }\end{array}$ \\
\hline
\end{tabular}

Fuente: Max-Neef. Desarrollo a escala humana. Disponible en: http://www.max-neef.cl/descargas/Max_Neef-Desarrollo_a_escala_humana.pdf 
Como se puede notar, la propuesta de desarrollo reflejada en la matriz anterior va enfocada no solo en el sentido del crecimiento económico de las sociedades, sino que implica la simbiosis de elementos personales, culturales, tecnológicos e incluso ambientales como lo señala Max-Neef:

La costumbre es utilizar indicadores con los que solemos pensar que el desarrollo en el que se crece más es mejor que en el que se crece menos. Sucede que esos indicadores nos dicen muy poco o casi nada. No nos dice la historia que 146 hay detrás (historias como la explotación laboral o la injusta distribución de la riqueza). (Max-Neef, 1993, citado por Berríos, 2007).

De tal forma, como indica Carvajal (2011),

lo interesante de este planteamiento es que nos lleva a una visión amplia de la llamada calidad de vida, teniendo en cuenta tanto las "necesidades existenciales" (ser, tener, hacer, relaciones), como las "necesidades axiológicas” (subsistencia, protección, afecto, comprensión, participación, creación, recreación, identidad y libertad).

En esa misma línea, la Teoría de la complejidad, según Alguacil
(2008, pp. 40 y 71), hace énfasis en la importancia de superar lo meramente cuantitativo para introducir también los aspectos cualitativos, a fin de cambiar la visión del bienestar por una perspectiva compleja de "calidad de vida”, que comporte la calidad ambiental (habitacional, residencial), el bienestar (trabajo, salud, educación) y la identidad cultural (tiempo libre, ocio, participación, relaciones y redes sociales).

Complementario de ello es que la calidad de vida se relaciona con aspectos no solo intrínsecos de los sujetos, sino que también comprende aspectos extrínsecos altamente importantes para las concepciones del desarrollo humano sostenible (PNUD, 1994, p. 15), en el entendido de que este valora la vida humana por encima de cualquier otro bien $\mathrm{y}$ tiene como objetivo permitir que todos los seres humanos tengan las mismas oportunidades, aumenten sus capacidades y las utilicen de la mejor forma posible, de modo que todo ello redunde en su propio beneficio y en el de los demás, así como conlleva también una seria preocupación por el cuidado de los recursos naturales en pro de las generaciones futuras.
Lo mencionado puede notarse con más claridad en el siguiente apartado que hace referencia a los intereses que busca el desarrollo humano sostenible:

Estas expectativas están relacionadas a una valoración de la vida más de tipo cualitativo que la población realiza (...). Lo anterior se refleja desde los ochenta del siglo pasado, en la posición de la Organización de Naciones Unidas (ONU) que se dirige a la búsqueda de la calidad en el desarrollo de las sociedades. Lo anterior va orientando los esfuerzos y el interés por nuevas visiones socioeconómicas que buscan la incorporación de la preocupación por el medio ambiente y la importancia de la subjetividad en el bienestar de los pueblos, fundamentándose en la búsqueda de sustentabilidad medio ambiental y social (Yasuko y Watanabe, 2011, p. 9).

Para Alguacil (2008, p. 40), el desarrollo humano sostenible tiene sentido solo en la búsqueda de la satisfacción de las necesidades humanas, regulada por los derechos humanos tanto de carácter individual como colectivo, y se circunscribe a la perspectiva de calidad de vida.

Por ello, las discusiones en torno al desarrollo ligado con la calidad de vida se han am- 
pliado, y deberán seguirse incrementando, por lo que cada vez van tomando más fuerza nuevas concepciones. En América Latina, por ejemplo, puede resaltarse a las comunidades indígenas de Ecuador y Bolivia que han presentado una propuesta diferente, que propende por lo que se ha denominado el Buen Vivir, entendido este como una crítica al desarrollo basado en la óptica meramente de crecimiento económico y visto más bien como una alternativa al propio desarrollo.

Así lo señala Gudynas (2012, pp. 50-51):

El buen vivir es una expresión que debe mucho a los saberes tradicionales, especialmente andinos. Sus referentes más conocidos son el sumak kawsay del kichwa ecuatoriano, y el suma qamaña del aymara boliviano. Pero no está restringido a ellos, y posturas similares se encuentran en otros pueblos indígenas, y algunas son de reciente configuración.

Entonces, el buen vivir lo que pretende es lograr la armonía entre los seres humanos, sus comunidades y la naturaleza o entorno, un apego total a los valores y derechos, que también se visibiliza en el caso colombia- no a través de las actividades y movimientos promovidos por sabedores indígenas del Amazonas y chamanes del Putumayo.

Consecuencia de estas nuevas visiones que se señalan, es que las constituciones políticas más recientes han reestructurado el concepto de desarrollo y van más allá de la calidad de vida, como ocurre con la Constitución Ecuatoriana de 2008, que contiene en el Capítulo Segundo los llamados Derechos del buen vivir, y que en voz de Gudynas y $\operatorname{Acosta}(2011$, p. 74) se refieren a: una amplia variedad de derechos (como a la alimentación, a un ambiente sano, al agua, a la comunicación, a la educación, a la vivienda, a la salud, a la energía, etc.). Por ejemplo, el artículo 14 "reconoce el derecho de la población a vivir en un ambiente sano y ecológicamente equilibrado, que garantice la sostenibilidad y el buen vivir, sumak kawsay.

Sin embargo, el mismo Gudynas explica que el buen vivir no consiste en regresar al pasado sino en construir futuro, y sirve de punto de encuentro a posturas éticas alternativas que le reconocen derechos a la naturaleza, e introducen nuevos conceptos de justicia y bienestar humano.
Finalmente, como lo señala Carvajal (2009, p. 15), hoy es necesario "hacer apuestas alternativas de desarrollo, incluyentes, con la multiplicidad -heterogeneidad- de actores", que coexistan en cada comunidad.

\section{Conclusiones}

- El desarrollo, en gran medida, ha dejado de relacionarse estrictamente con el crecimiento económico de los países, para ampliar su espectro incluyendo elementos que son igualmente importantes, como las capacidades humanas, el entorno, la cultura e incluso el medio ambiente, lo que ha permitido que se hable de desarrollo humano y de desarrollo humano sostenible.

- Debido a la multiplicidad de factores y elementos que inciden en el desarrollo, las posturas de pensamiento actuales abogan por una visión multidimensional del mismo que abarque lo humano, lo económico, lo ecológico y lo social.

- Por su parte, la calidad de vida está íntimamente ligada con una serie de factores tanto de carácter intrínseco 
como extrínseco de los sujetos y no puede de ninguna manera limitarse a la medición desde el ámbito económico.

- La calidad de vida ha sido una preocupación constante de los gobiernos. En aras del mejoramiento de esta se han producido políticas públicas que, como en el caso de Colombia, han resultado insuficientes e inoperantes pues su formulación se ha enfocado inadecuadamente y desconoce las realidades de los individuos.

148 - Retomando las palabras de Carvajal (2009, p. 16), las nuevas sociedades deben apostarle a los pensamientos alternativos que no solo se pueden hacer desde el ámbito local, sino que tienden cada vez más a ser globales. Así, definitivamente, como se ha expresado en los foros sociales mundiales, otro mundo y mejor sí es posible.

\section{Referencias}

Alguacil, J. (2008). El desarrollo social hoy: caminando hacia el desarrollo humano sostenible. Madrid: Universidad Carlos III de Madrid. Recuperado de http://habitat.aq.upm. es/boletin/n50/n50-ajalg. pdf

Berríos, O. (2007). El desarrollo a escala humana de Manfred Max-Neef. En La broma.org. Recuperado de http://www.labroma.org/ blog/2007/01/21/el-desarrollo-a-escala-humana/

Carvajal, A. (2011). Desarrollo y cultura. Elementos para la reflexión y la acción. (3 ed.). Cali: Escuela de Trabajo Social y Desarrollo Humano - Universidad del Valle. Recuperado de http:/ / desarrollolocalteoriaypractica. blogspot.com/2011/04/ desarrollo-y-cultura.html

Carvajal, A. (2009). ¿Modelos alternativos de desarrollo o modelos alternativos al desarrollo? Revista Prospectiva, 14. Recuperado de http:/ / dintev.univalle.edu. co/revistasunivalle/index. $\mathrm{php} / \mathrm{prospectiva/article/}$ view/355

DE NEGRI, A. (2002). Estrategia de promoción de la calidad de vida. Caracas: Agencia de Cooperación Técnica Alemana, Ministerio de Salud y Desarrollo Social de la República Bolivariana de Venezuela.

Departamento Nacional de Es- tadística -DANE. Encuesta Nacional de Calidad de Vida 2011 y 2010. Recuperado de http//www.dane.gov.co/ files/investigaciones/condiciones_vida/calidad_vida/ cp_ECV_2011.pdf

DEPARTAMENTO NACIONAL DE Estadística - DANE. Análisis de la Encuesta Nacional de Calidad de Vida. Recuperado de:http:// 190.25.231.249/ aplicativos/sen/NADA/ ddibrowser $/$ ?id $=108$

GamboA, L. F., Cortés, D. y GonzÁlez, J. (2000). Algunas consideraciones analíticas sobre el estándar de vida. Recuperado de http://revistas.urosario.edu.co/index.php/economia/article/ view/993/892

Gudynas, E. y Acosta, A. (2011). El buen vivir más allá del desarrollo. Recuperado de http://www.gudynas.com/ publicaciones/reportes/ GudynasAcostaBuenVivirDesarrolloQHacer 11.pdf

Gudynas, E. (2012). Debates sobre el desarrollo y sus alternativas en América Latina: una breve guía heterodoxa. En Más allá del desarrollo (pp. 50-51). Cali: Merlín I. D.

KAlipedia. (2008). Condiciones de vida en Colombia. Recupe- 
rado de http://co.kalipedia. com/geografia-colombia/ tema/geografia-economica-social/_-indice-condiciones-vida-colombia. html?x=-2008080 1 klpgeogco-_29.Kes\&ap=1

LAMPIS, A. (2007). Vulnerabilidad y protección social en Colombia: estudios de caso en Bogotá, Cali y Manizales. En: C. Zorro (Com.). El desarrollo: perspectivas y dimensiones. Aportes interdisciplinarios. Bogotá: Universidad de los Andes.

Nussbaum, M. (2012). Las mujeres y el desarrollo humano. Barcelona: Herder Editorial. Nussbaum, M. y SEn, A. (2001). Calidad de vida. En J. Montesino. Reseña de la calidad de vida (pp. 2-3). Recuperado de http://redalyc.uaemex. $\mathrm{mx} / \mathrm{pdf} / 305 / 30500231$. pdf
Palomba, R. (2009). Calidad de vida: conceptos y medidas. Taller sobre calidad de vida y redes de apoyo de las personas adultas mayores. Santiago de Chile : CELADE-CEPAL.. Recuperado de http://www.eclac.cl/ celade/agenda/2/10592/ envejecimientorp 1_ppt.pdf

PRogRama de Las NaCiONeS

UNIDAS PARA EL DESARROLLO. Informe sobre desarrollo humano 1994. Hacia el desarrollo humano sostenible. Nueva York: Fondo de Cultura Económica S. A. de C. V.

PROGRAMA DE LAS NACIONES UNIDAS PARA EL DESARROLLO. Informe sobre desarrollo humano 2013. Un Sur más global. Nueva York: Fondo de Cultura Económica S. A. de C. V.
Sarmiento, A. (2010). La multidimensionalidad en Colombia. En: Seminario Internacional: Colombia en las nuevas tendencias de medición de pobreza e igualdad de oportunidades. Bogotá. Recuperado de http://www. dnp.gov.co/LinkClick.aspx? fileticket $=\mathrm{QEZB} 3 \mathrm{EoY} 8 \mathrm{~F}_{\mathrm{w}}$ $\% 3$ D\&tabid $=1192$

SEn, A. (2000). Desarrollo y libertad. Barcelona: Planeta.

Yasuko, B., Watanabe, A. (2011). La calidad de vida: eje del bienestar y el desarrollo sostenible. Revista Hologramática, VII, 1 (15), 149 p. 9. Recuperado de http// www.hologramatica.com.ar o www.unlz.edu.ar/sociales/hologramatica

Yunus, M. (1998). Hacia un mundo sin pobreza. Barcelona: Andrés Bello. 